\title{
Liver disease progression in HIV/HCV co-infected patients: a role for metalloproteinases and their specific inhibitors
}

\section{Priyo Sasongko*}

\author{
Address: Government, Indonesia
}

* Corresponding author

\author{
from 2006 International Meeting of The Institute of Human Virology \\ Baltimore, USA. 17-21 November, 2006 \\ Published: 21 December 2006 \\ Retrovirology 2006, 3(Suppl I):P58 doi:I0.I 186/I742-4690-3-SI-P58
}

(c) 2006 Sasongko; licensee BioMed Central Ltd.

\section{Background of Study}

$\mathrm{HIV}$ infection accelerates the rate of $\mathrm{HCV}$ progression to fibrosis which represents the main factor affecting the prognosis of hepatitis $\mathrm{C}$ as well the best indicator of disease status. There is increasing evidence that liver fibrosis is a dynamic pathology c process in which the altered balance between matrix metalloproteiases (MMPs) and their specific inhibitors (TIMPs) may play a major role.

\section{Objective of the Study}

To investigate the possible involvement of MMP-9 and TIMP-1 in the HCV liver disease progression in patients co-infected with HIV, we assessed the levels of circulating enzyme and inhibitor in a series of HIV-infected individuals with and without chronic hepatitis $\mathrm{C}$.

\section{Design}

Study participants included a total of 76 HIV-infected patients, of whom 49 without HCV infection (median CD4 $=241 / \mathrm{mmc} ; \mathrm{VL}=5 \mathrm{log}$ ) and 27 co-infected with HCV (median CD4 = 130/mmc; VL = $5.3 \mathrm{log}$ ). All but one of $\mathrm{HIV} / \mathrm{HCV}$ co-infected patients had evidence of chronic hepatitis C. 11 healthy donors were used as controls. Concentrations (ng/ml) of human TIMP-1 and MMP-9 were detected in plasma samples using the Biotrak ELISA assay (Amersham). Data are expressed as median.

\section{Results}

All HIV-infected patients had plasma TIMP-1 levels significantly higher than healthy controls (1740 vs. 755), whereas MMP-9 levels were lower (23 vs. 1157) ( $\mathrm{p}<$ $0.001)$. The levels of TIMP-1 were significantly higher in patients with CD4 > 300/mmc than those with CD4 < $300 / \mathrm{mmc}(\mathrm{p}<0.05)$. No statistically significant differences in the levels of MMP-9 and in the TIMP-1/MMP-9 ratio were found between $\mathrm{HCV}$ co-infected and not coinfected HIV+ patients $(\mathrm{p}<0.05)$.

\section{Conclusion}

Our results suggest that the altered balance between MMP-9 and TIMP-1 during HIV infection may play an important role in exacerbating fibrosis progression in patients co-infected with HCV. 